\title{
PROSODIC VARIATION AND AUDIENCE RESPONSE
}

\author{
Marian Shapley
}

\section{Introduction}

While variation in speakers' voices has been well studied for short stretches of speech, such as clauses and sentences, less work has been reported on the prosody of longer periods of speech of different types. This paper describes some aspects of prosodic style of speakers during an entire narrative in a conversational discourse and relates the style to the amount of response received from the listeners. Since neither "discourse mode" nor "prosody" are well-defined concepts, their meaning for this paper will be briefly outlined.

\subsection{Prosody.}

The term "prosody" as used here is equivalent to supra-segmentals, which are "those aspects of speech which involve more than single consonants or vowels" (Ladefoged 1975). The primary prosodic features include pitch, loudness and length; voice quality is another feature. Pitch and speaking rate are used in this study.

\subsection{Discourse Mode.}

Discourse mode may be described along several dimensions, including the social context in which the speech takes place, such as classroom situations, public addresses, telephone conversations, the relations between the participants, the amount of preparedness or familiarity with the material, such as in prepared lectures, reading material aloud, vs. telling stories or spontaneous conversation , and also the task set for the speaker in experiments, such as reading different materials or simulating various emotions. Johns-Lewis (1986b) suggests as starting bases for intonation taxonomies the spontaneous/non-spontaneous distinction, the public/private dimension and the relative status and expertise of speaker vis-a-vis audience. 
As there is as yet no comprehensive classification of discourse modes, the name given to a discourse mode by a researcher is usually descriptive of the setting of the speech, such as "political interview" or "reading aloud" and the dimensions involved usually can be inferred from this name.

\subsection{Studies of discourse modes and prosodic styles.}

Most studies of prosodic style compare styles either in terms of timing data, such as rate of speech and occurrence of pauses, or of pitch data, such as average pitch, range, and shape of the pitch distribution.

Studies of discourse mode involving speaking rate have shown that rate of speech is slower in telling a story than reading it aloud (Levin et al. 1982), slower in casual interviews than in political speeches and political interviews (Duez 1982), and also slower in spontaneous speech than in prepared or semi-prepared material (Barik 1979). While the methodologies of these studies is not always consistent, for example Duez's and Barik's definitions of pauses differ, and they used different speakers in different modes, still the results agree in the sense that the less formal, less planned modes are associated with slower rates.

In a study investigating the pitch characteristics of discourse, Johns-Lewis (1986b), investigated three discourse modes: reading a monologue as though acting, reading a narrative, and spontaneous conversation. She found that the acting mode had the highest pitch and conversation the lowest. Graddol (1986), comparing differences due to content of reading materials, found that subjects had a higher average pitch when reading a dialogue than when reading a technical manual. Fernald and Simon (1984), studying "motherese" in German women, found a higher pitch for mothers talking to their babies than for talking to adults. These studies all started with given discourse modes such as "reading aloud" or "conversation" and compared their prosodic variables.

While prosodic features can be related to such discourse modes, prosody may vary greatly within a mode. A single speaker in a single speech situation such as casual conversation may make use of various styles. The 
present study, using narratives in conversations, contrasts styles of the same speaker within the same speech event, and speech modes are defined in terms of amount of listener response. This method has the advantage of controlling variability among speakers and situations. That is, sociological factors such as relationships of speaker to listener, and expertise of speaker in the mode are constant. What varies is the prosodic style, and these styles can be defined in terms of what is occurring in the speech situation.

\section{Data and Methodology.}

The data consist of two narrations taken from recorded multi-person conversations. There is one male speaker, Mark, and one female, Fern. The narrations have semantic and syntactic continuity, and contain the turn structures typical of stories, ${ }^{1}$ with the listeners occasionally participating in the talk.

\subsection{Measurement of audience response.}

The independent variable of this comparison is called audience response. The texts were categorized as High response when they were accompanied by many responses, and Low response when accompanied by few responses during the narration. This does not mean that the amount of response is a categorical variable, but only that categories form a convenient way of contrasting extremes. Responses were noted both between articulated portions of speech and during speaking. Thus speaking by someone other than the narrator, whether a successful turn such as a question or a feedback response like "uh huh", or an attempted turn, was a response. Laughter either during or between speech groups, was called a response. While the word "audience" usually refers to listeners and not to the speaker, in this case the speaker's own response to what he is saying is included. That is, during the telling of something a speaker will often laugh once, or even several times in mid-utterance, without indication of pausing, but rather including a laugh token as another word. And the speaker may also laugh following his own utterance, creating a laugh response between speech intervals. These situations were called self-responses. So audience in this data includes the speaker. 2 
The following scheme describes the possible responses.

Responses when narrator is not talking:

By audience: with words or feedback tokens

(a turn)

with laughter

By speaker: with laughter

By both: with laughter

Responses during talking:

By audience: speaking while talking is going on (an attempted turn)

with laughter

By speaker: with laughter

By both: with laughter

A period of 25 seconds was taken as a unit for measuring the amount of response. A moving total of the number of responses in this window was computed, and when the total remained at 5 or above $(0.2$ responses per second or a response every 5 seconds), the speech was classed as High mode, (with much audience response); when it was below this figure, it was classed as Low mode (with little audience response). By this criterion each narration was split into two sections or sub-modes., hereafter referred to as modes. The responses per second for the speaker-modes, are listed in Table 1.

Speaker

Mark

Fern
Responses per Second

Low Mode High Mode

.04

.08
.33

.33

Table 1. Responses per second by mode assigned

More information about the kinds of responses is shown in Tables 2 
and 3. In Table 2, actual turns at talk are differentiated from laughter as a response. There are many more laughter responses than verbal responses, and laughter differentiates the modes better than talk responses.

$$
\begin{array}{lll}
\text { Responses per second } & \text { Total } \\
\text { Talk } & \text { Laughter } & \text { responses }
\end{array}
$$

Low Mode

$\begin{array}{llll}\text { Mark } & 0.01 & 0.03 & 4 \\ \text { Fern } & 0.05 & 0.02 & 6\end{array}$

High Mode

$\begin{array}{llll}\text { Mark } & 0.07 & 0.27 & 25 \\ \text { Fern } & 0.04 & 0.28 & 46\end{array}$

$\begin{array}{llll}\text { Total responses } & 16 & 65 & 81\end{array}$

Table 2. Turns and laughter as responses

Listener responses are compared with self-responses In Table 3. Listener responses predominate here, with more in High mode than Low mode.

\begin{tabular}{lcc} 
& \multicolumn{2}{c}{ Responses per Second } \\
By listener & Self-response \\
Low Mode & & \\
Mark & 0.03 & 0.01 \\
Fern & 0.08 & - \\
High Mode & & \\
$\quad$ Mark & 0.28 & 0.03 \\
$\quad$ Fern & 0.24 & 0.08 \\
Total responses & 67 & 14
\end{tabular}

Table 3. Listener responses vs. self-responses

\subsection{Pitch data.}

The data was recorded in non-laboratory situations, in some cases 
with considerable background noise, but nevertheless, reasonable pitch data could be determined. Pitch was recorded in the form of fundamental frequencies derived from spectrograms. While pitch, a perceptual phenomenon, and fundamental frequency, an acoustic phenomenon, are not measured in equivalent steps, the relation is close enough so that fundamental frequency can be used as an estimate of pitch. Data were recorded for every tenth of a second, as fundamental frequency values, or pauses.

The pitch variables for each speaker-mode include the mean, median, range, variation, skew and peakedness (kurtosis) of the pitch distribution ${ }^{3}$. Skew, a measure of the extent to which the values fall on one side of the mean score or the other, and peakednesss, a measure of how peaked or flat the distribution is in comparison with a normal distribution, are measures which have been used to distinguish prosodic style.

\subsection{Timing data.}

The number of pauses was totalled for each mode, as was the number of syllables. A pause was counted when a period of at least 0.3 seconds without speech occurred. More pauses occurred in the Low mode than in the High mode, and they were longer in the Low mode than in the High mode.

$\begin{array}{lcl}\begin{array}{l}\text { Speaker } \\ \text { \& Mode }\end{array} & \begin{array}{l}\text { Number of } \\ \text { pauses }\end{array} & \begin{array}{l}\text { Average } \\ \text { length }\end{array} \\ \text { Mark/Low } & 41 & 5.92 \\ \text { Mark/High } & 9 & 4.78 \\ & & \\ \text { Fern/Low } & 35 & 7.51 \\ \text { Fern/High } & 20 & 5.55\end{array}$

Table 4. Number of pauses and average length in seconds 
3. Results.

Figure 1 shows graphically the distinction between the High and Low modes. The pitch in $\mathrm{Z}$-scores ${ }^{4}$ is plotted by time for both narratives.

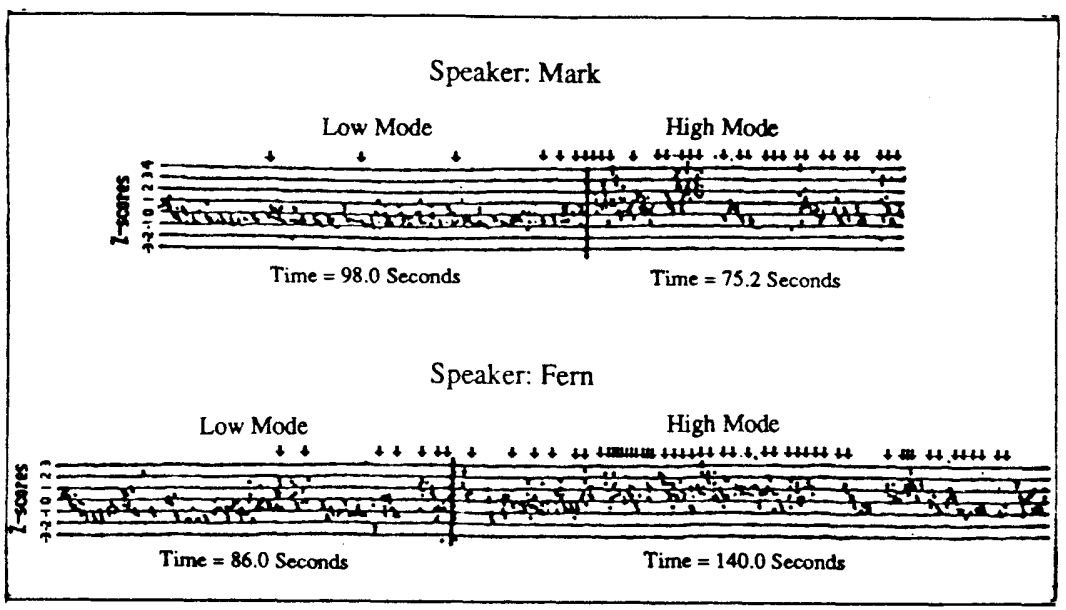

Figure 1. Pitch values by time for speaker-modes. Dots represent fundamental frequency values in $\log \mathrm{Z}$-scores on the vertical scale, plotted against time in $1 / 10$ th second intervals on the horizontal scale. Arrows indicate times of audience response.

\subsection{Pitch Comparisons.}

The pitches of the High response and the Low response modes differ from each other in that the High modes have higher mean and median pitches. These differences are significant for intra-speaker comparisons at the .001 level. 
The upper and lower bounds of pitch were taken here to be the 5th and 95 th percentiles. This excludes outliers; this measure has been used by other researchers because it was considered more representative of the functional range of speakers than the difference between the absolute highest and lowest pitches. These maximum and minimum values also discriminated between modes for both speakers.

$\begin{array}{llllll}\begin{array}{l}\text { Speaker } \\ \text { \& Mode }\end{array} & \text { Mean } & \text { Median } & \begin{array}{l}\text { 5th Per- } \\ \text { centile }\end{array} & \begin{array}{l}\text { 95th Per- } \\ \text { centile }\end{array} & \text { N } \\ & & & & & \\ \text { Mark/Low } & 32.6 & 32.0 & 27.4 & 38.4 & 665 \\ \text { Mark/High } & 37.8 & 37.2 & 29.5 & 47.9 & 412 \\ & & & & & \\ \text { Fern/Low } & 42.9 & 42.4 & 38.4 & 49.7 & 521 \\ \text { Fern/High } & 46.5 & 46.5 & 41.5 & 53.2 & 745\end{array}$

Table 5. Pitch measures of central tendency

$\begin{array}{lcccc}\begin{array}{l}\text { Speaker } \\ \text { \& Mode }\end{array} & \begin{array}{l}\text { Standard } \\ \text { Deviation }\end{array} & \text { Range } & \text { Skew } & \begin{array}{l}\text { Peaked- } \\ \text { ness }\end{array} \\ \text { Mark/Low } & 2.9 & 11.0 & .56 & .63 \\ \text { Mark/High } & 5.5 & 18.4 & .55 & .43 \\ & & & & \\ \text { Fern/Low } & 3.6 & 11.3 & .78 & .08 \\ \text { Fern/High } & 3.8 & 11.7 & .01 & .38\end{array}$

Table 6. Pitch measures of dispersion and skewness

The measures describing the variation and shape of the pitch distribution did not in general discriminate modes consistently for both speakers. While the standard deviations and the ranges did differ in a consistent direction for each speaker, for Fern the difference was small. 
Skewness and peakedness measures were inconsistent between the speakers. The shape of the pitch distribution may be an individual phenomenon, a reflection of how much of their range individual speakers use.

\subsection{Speech Rate.}

Two rates of speech were computed: the speaking rate, which measures syllables per second of elapsed time, and the articulation rate, which measures the syllables per second excluding pauses. Both speakers had slower rates in the High mode, by both measures.

$\begin{array}{lcc}\begin{array}{l}\text { Speaker } \\ \text { \& Mode }\end{array} & \begin{array}{c}\text { Articulation } \\ \text { rate }\end{array} & \begin{array}{c}\text { Speaking } \\ \text { rate }\end{array} \\ \text { Mark/Low } & 5.7 & 4.2 \\ \text { Mark/High } & 4.2 & 3.9 \\ & & \\ \text { Fern/Low } & 6.5 & 4.4 \\ \text { Fern/High } & 4.7 & 3.9\end{array}$

Table 7. Rate of speaking in syllables per second

\section{Discussion.}

The categories of High audience response and Low audience response do not fit neatly into the taxonomy suggested by Johns-Lewis. However if one considers the High mode to reflect more spontaneity, then the rates of speaking found here are comparable to those found by other researchers, that is, the more spontaneous the speech, the slower it is. While the larger number of pauses in the Low categories (seen in Table 4) would seem to have the effect of slowing the speech rate, in actuality they were balanced by the larger number of laughter responses in the High categories, which entered into the rate computation. 
On the other hand, the pitch data, which show a higher pitch for the more spontaneous mode in this sense, disagree with that of Johns-Lewis, who found the lowest pitch for conversation. Graddol's result of higher pitch for reading dialogue than for reading a technical manual is not really comparable to the present data. While pitch differences do reflect the content of speech, as Graddol claims, and the discourse mode as Johns-Lewis claims, it is likely that the noted difference between styles is due to some common factors in the situations.

When the difference in two modes of speech is expressed in terms of a single pitch figure, such as a mean, it can be interpreted as a difference in register, that is, a raised or lowered pitch level throughout the excerpt. This sustained pitch level over a period of several clauses has also been correlated with the expression of emotions (e.g. Fonagy 1978, Scherer 1977, Williams and Stevens 1972). The statistics for the data in the above discourse modes of continuous speech have the same kind of scope, and may as well be measuring "joy" or "sorrow", as "reading dialogue".

That is, differences in style may reflect differences in personal involvement. It is therefore not surprising that the results of studies of pitch and discourse style are sometimes inconsistent, since involvement is not always a function of the type of discourse, although it often may be. There are those who can read a phone book, or the alphabet, with great emotional style, but it is more commonly the case that such prosodic delivery is limited to speech in which the speaker is involved, or to acting. The real question is then to discover the linguistic or situational cues to this involvement, and how such cues affect pitch level. The dialogue situation seems to be one, and audience response (or lack of it) is a part of real dialogue, and should be counted among the cues to change of pitch.

In the case of narrations, audience response may itself be related to the structure of the talk. For example, in telling a story there are parts which are more likely to be expressed with emotion than others. Labov (1972), describes six possible elements of a story: abstract, orientation, complicating action (events told in temporal order), evaluation, results or resolution and a coda, which brings the talk back to the current situation. The evaluation 
element gives tellers' reactions and opinions about the story, and it is largely in this type of talk in the anecdotes where the style differs, and also where the audience response occurs. The style difference therefore may be as much a function of the story structure as of the response.

There are problems in dealing with distributional descriptions of pitch, that is describing a discourse mode by a single figure. Such measures are obviously sensitive to the measures used to describe the data, such as the interval and the rate of responses decided upon, and information about the shape of the distribution may be obscured. As can be seen in Figure 1, the selection of a different interval of measurement could result in a different segmentation of the talk into modes, and possibly in different prosodic measurements. A finer categorization which could be related to the content of the talk might prove fruitful.

\section{Conclusions.}

Differences in prosodic style can covary with the interactional factor of audience response. Differences such as those found between discourse modes, and between types of textual material, also occur in a single, conversational discourse mode, in a single story-telling.

The categorization of conversational speech into a high audience response mode and a low audience response mode is independently motivated by the prosodic variables of pitch level and speaking rates. The style difference is interpreted as a reflection of a more basic variable such as the amount speaker involvement. The actual correlates of this kind of involvement are still to be determined.

This paper is based on data from only two speakers and of course more data is needed. Since it is based on naturally occurring speech, it shows a relationship which can occur, but does not show that the relationship need necessarily occur. 


\section{NOTES}

${ }^{1}$ For a description of turns and turn-taking see Sacks, Schegloff and Jefferson (1974). For turns in story structure see Jefferson (1978).

2 In the face-to-face situation in which the data was recorded there are bound to be nonverbal responses such as facial and body gestures, which may also play an important role. However, the data was not available.

${ }^{3}$ The pitch values are given in a semi-tone (octave) scale.

$4 \mathrm{Z}$-scores are computed by converting the mean of a distribution to zero, and assigning a score to each value in terms of the number of standard deviations a value is from this mean of zero. 


\section{REFERENCES}

Barik, H. C. (1979) Cross-linguistic study of temporal characteristics of different types of speech materials. Language and Speech 20: 116-126.

Duez, D. (1982). Silent and non-silent pauses in three speech styles. Language and Speech 25: 11-28.

Fonagy, I. (1978) A new method of investigating the perception of prosodic features. Language and Speech 21: 34-49.

Fernald, Anne and Thomas Simon (1984) Affective and perceptive saliences in mother's speech. Developmental Psychology 20: 104-114.

Graddol, David. Discourse specific pitch behavior. In Johns-Lewis 1986a.

Jefferson, Gail. Sequential aspects of storytelling. In Schenkein, Jim (1978) Studies in the Organization of Conversational Interaction. New York: Academic Press.

Johns-Lewis, Catherine M. (1986a). Intonation in Discourse. London: Croom Helm

Johns-Lewis, Catherine M. (1986b). Prosodic Differentiation of discourse modes. In Johns-Lewis (1986), pp.199-219.

Labov, W.(1972). Language in the Inner City: Studies in Black English Vernacular. Philadelphia: University of Pennsylvania Press.

Ladefoged, Peter (1975). A Course in Phonetics. New York: Harcourt, Brace Jovanovich.

Levin, H., C.A. Schaffer and C. Snow (1982). The prosodic and paralinguistic features of reading and telling stories. Language and Speech 25: 43-54. 
Sacks, Harvey, E. A. Schegloff and G. Jefferson (1974). A simplest systematics for the organization of turn-taking for conversation. Language 50: 696-735.

Scherer, K. R. (1977). The effect of stress on the fundamental frequency of the voice. L.Acoustical Soc. Amer. 62: Supplement 1, 25-6 (abstract). [Cited in Scherer, Klaus R. (1979) Personality markers in speech. In Klaus R. Scherer and Howard Giles (eds.) Social markers in speech. Cambridge: University Press

Williams, Carl E. and Kenneth Stevens (1972). Emotions and speech: some acoustical correlates. J.Acoustical Soc. Amer. 52: 1238-1256. 